\section{Comparison of 25- and 23-gauge sutureless microincision vitrectomy surgery in the treatment of various vitreoretinal diseases}

Y Nam, H Chung, JY Lee, JG Kim and YH Yoon

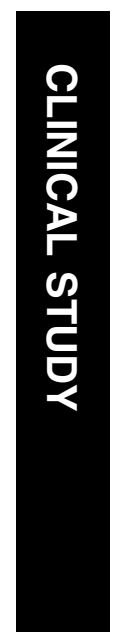

thus may be made according to a surgeon's preference and the availability of appropriate instruments.

Eye (2010) 24, 869-874; doi:10.1038/eye.2009.206;

published online 14 August 2009

Keywords: microincision vitrectomy surgery; minimally invasive; sutureless vitrectomy

\section{Introduction}

Modern vitrectomy had been performed using 20-gauge instruments for $>30$ years before 2002 when Fujii and co-workers introduced the first commercially available sutureless

transconjunctival vitrectomy technique using a 25-gauge instrument. ${ }^{1-3}$ The incision is small and a suture is not required, so sutureless 25gauge microincision vitrectomy surgery (MIVS) has enabled minimization of surgical trauma and suture-related inflammation. MIVS shortens operative time and facilitates postoperative recovery. ${ }^{4-12}$

This 25-gauge sutureless system, however, was useful only in cases of relatively simple vitreoretinal pathology owing to limitations in cutter performance and a lack of necessary accessory instruments. In 2005, Eckardt developed 23-gauge sutureless vitrectomy using an obliquely entering trocar cannular system. ${ }^{13}$ Although the 23-gauge system seemed to offer improvements in cutter efficiency and rigidity, it was thought that the larger sclerotomy size was likely to increase the risk of wound-related problems.

In the past few years, advances in microincision vitrectomy techniques, in both the
Department of Ophthalmology, Asan Medical Center, University of Ulsan, College of Medicine, Seoul, Korea

Correspondence: YH Yoon, Department of Ophthalmology, Asan Medical Center, 388-1 Pungnab-dong,

Seoul 138-736,

Korea

Tel: + 82230103675 ;

Fax: + 8224706440 .

E-mail:yhyoon@

amc.seoul.kr

Received: 17 December 2008

Accepted in revised form: 6 July 2009

Published online: 14 August 2009

This work was supported by a grant of the Korea Healthcare of Technology R\&D Project, Ministry of Health, Welfare \& Family Affairs, Republic of Korea (\#A080557) (Dr Yoon). Songpa-gu, 
25- and 23-gauge systems, have been remarkable in terms of cutter efficiency, illumination technology, and the development of various microsurgical accessories. Although a few studies have compared these minimally invasive MIVS approaches with conventional 20-gauge vitrectomy, ${ }^{6,7,14-17}$ no comparison between 25 - and 23-gauge MIVS has yet been reported.

In this prospective study, we compared the surgical outcomes and complications of the two MIVS techniques used in the management of various vitreoretinal diseases.

\section{Patients and methods}

A prospective, comparative, and randomized clinical trial was performed in 85 consecutive patients undergoing transconjunctival MIVS at a single academic centre between January and April 2008. This study was approved by the Institutional Review Board of the Asan Medical Center. Patients were given full information on the study, told of the benefits and risks, and gave written consent before surgery.

Included were patients with preoperative diagnoses of vitreous haemorrhage (either diabetic or non-diabetic), diabetic traction retinal detachment classed as moderate or below, cystoid macular oedema, macular pucker, full-thickness macular hole, vitreous opacities, rhegmatogenous retinal detachment, and other less-complicated vitreoretinal pathologies.

Patients were randomly allocated to either 25- or 23-gauge MIVS using a table of random numbers. Thirtyeight patients were treated with the 25-gauge technique and 47 with the 23-gauge technique. All procedures were conducted by one surgeon (YHY) using the Accurus vitrectomy system (Alcon, Fort Worth, TX, USA) under a retrobulbar block and using a 50:50 (v/v) mixture of $2 \%$ $(\mathrm{w} / \mathrm{v})$ lidocaine and $4 \%(\mathrm{w} / \mathrm{v})$ bupivacaine. Cutter speed and aspiration pressure for 25 and 23 gauges were $1500 \mathrm{cpm}$ with $550 \mathrm{~mm} \mathrm{Hg}$ and $2500 \mathrm{cpm}$ with $350 \mathrm{~mm} \mathrm{Hg}$, respectively.

Surgical procedures were basically similar in both groups, except for the scleral-entry procedure. In 25-gauge MIVS, after displacing the conjunctiva above the intended sclerotomy site, a disposable one-step 25-gauge trocar with a microcannula was inserted perpendicularly into the midvitreous. In 23-gauge MIVS, after displacing the conjunctiva, a $15-30^{\circ}$ angled tunnel insertion was made using a disposable one-step 23-gauge trocar and a microcannula. For those eyes that underwent combined cataract surgery, phacoemulsification and intraocular lens implantation were performed with the inferotemporal trocar in place. Complete removal of the vitreous gel was performed, after inducing posterior vitreous detachment when necessary. For tractional retinal detachment cases, a vitreous cutter was used to shave the residual membrane stump after delamination or segmentation with microscissors. When a macular hole was present, and in cases of macular pucker and diabetic macular oedema, indocyanine green dye was used to enhance visualization of the internal limiting membrane. In cases of macular hole, myopic foveoschisis, and retinal detachment, a 6-10\% (v/v) C3F8 gas tamponade was performed. In eyes with macula off-tractional detachment, severe macular oedema, or preoperative wrinkling, a small bubble of air was injected to fill $20-30 \%$ of the total vitreous cavity to facilitate postopereative resolution of macula oedema or wrinkling. ${ }^{18}$ Surgery was completed by removal of the entry site alignment cannulas without suturing the conjunctiva and sclera. If any site showed persistent leakage, the conjunctiva was opened and 7-0 vicryl sutures were placed in the wound and the overlying conjunctiva.

Preoperative data included patient age, gender, best-corrected visual acuity (BCVA), intraocular pressure (IOP), biomicroscopic findings, and detailed fundoscopic information. When needed, b-scan echography, fluorescein angiography, and optical coherence tomography were also performed. During operation, the operation time and amount of balanced salts solution (BSS) consumed were measured. Postoperative BCVA and IOP were measured at 1 day, 1 week, and 1 and 3 months after surgery. As a substitute for a patient survey on the postoperative discomfort, the degrees of postoperative conjunctival and corneal change were qualitatively graded using an earlier reported standardized scale ${ }^{19,20}$ with some modifications. Briefly, each category of conjunctival injection, conjunctival oedema, subconjunctival haemorrhage, and corneal oedema were scored by slit-lamp examination as 0 (absent), 0.5 (trace), 1 (mild), 2 (moderate), and 3 (severe). Intraoperative and postoperative complications such as sclerotomy site wound leakage, hypotony as shown by IOP $<8 \mathrm{~mm} \mathrm{Hg}$, retinal tear, retinal detachment, or endophthalmitis were all recorded.

\section{Statistical analysis}

Snellen visual acuity data were converted into logarithms of the minimum angle of resolution for statistical analysis. Independent $t$-test was used to compare operation times and amounts of BSS consumed. The relationship between preoperative and postoperative visual acuity was statistically analysed using the independent $t$-test. A $P$-value $<0.05$ was defined as statistically significant. 


\section{Results}

As shown in Table 1, there were no statistically significant differences in patient characteristics or preoperative diagnoses between the 25- and 23-gauge groups.

The mean duration of vitrectomy (in combined surgical cases, cataract surgical time was excluded) was $33.7 \mathrm{~min}$ (range 15-64 min) in the 25-gauge group and $34.5 \mathrm{~min}$ (range 25-63 min) in the 23-gauge group $(P=0.942)$. In the 25-gauge MIVS group, the average amount of BSS consumed was $55.3 \mathrm{ml}$ (range 30-150 ml) and in the 23-gauge group, it was $72.3 \mathrm{ml}$ (range 15-220 ml) $(P=0.002)$.

Figure 1 shows a comparison of preoperative visual acuity and postoperative visual acuity recovery between all patients of the 25- and 23-gauge MIVS groups. Although each group showed a significant improvement in postoperative visual recovery at 1 and 3 months, there was no statistically significant difference between the two groups at any follow-up time. Interestingly, the patterns of visual recovery were similar in all groups when we categorized total eyes into four subgroups according to preoperative diagnosis (Figure 2). Therefore, we conclude that the size difference between 25 - and 23-gauge cutters did not affect the speed of postoperative visual recovery.

The postoperative anterior segment scores are shown in Table 2. Whereas most parameters suggested that the 25-gauge MIVS eyes had undergone less trauma, only conjunctival oedema was significantly less at 1 week in the 25-gauge MIVS group compared with the 23-gauge group.

As shown in Table 3, intraoperatively, suturing of sclerotomy sites was required for three eyes in the 25-gauge group and seven in the 23-gauge group. Retinal tears were created during surgery in one patient of each group, but were treated without difficulty. Two 25-gauge patients (air bubble filled) and three 23-gauge patients ( 1 fluid filled, 1 air bubble filled, and 1 silicone oil filled) showed IOP of $<8 \mathrm{~mm} \mathrm{Hg}$ at postoperative 1 day. However, all five patients recovered normal IOP without additional suturing or other procedures at 1 week postoperatively. No patient in either group developed wound leakage, retinal detachment, choroidal detachment, or endophthalmitis.

\section{Discussion}

Sutureless MIVS has increased in popularity owing to short operation time, less patient discomfort, and faster visual recovery. ${ }^{3-12}$ Furthermore, a few recent reports continue to prove the superiority of MIVS in direct comparison with conventional 20-gauge vitrectomy. $6,7,14-17$
Table 1 Patient characteristics

\begin{tabular}{lcc}
\hline & MIVS25 & MIVS23 \\
\hline Number of eyes & 38 & 47 \\
Mean age (years \pm SD) & $58.3 \pm 12.4$ & $59.1 \pm 11.0$ \\
Sex (male/female) & $15 / 23$ & $23 / 24$ \\
Diagnosis & & \\
VH & 14 & 20 \\
DMTRD & 2 & 5 \\
ERM & 11 & 10 \\
CME & 5 & 0 \\
FTMH & 3 & 5 \\
Others & 3 & 7 \\
Retinal detachment & 0 & 2 \\
Macular schisis & 0 & 3 \\
Vitreous opacity & 3 & 2 \\
Phaco/IOL & $27 / 38$ & $38 / 47$ \\
Air/gas tamponade & $20 / 4$ & $25 / 12$
\end{tabular}

Anaesthesia

Retrobulbar

$38 / 38$

$47 / 47$

CME, cystoid macular oedema; DMTRD, diabetic traction retinal detachment; ERM, epiretinal membrane; FTMH, full-thickness macular hole; Phaco/IOL, phacoemulsification and intraocular lens implantation; $\mathrm{SD}$, standard deviation; $\mathrm{VH}$, vitreous haemorrhage.

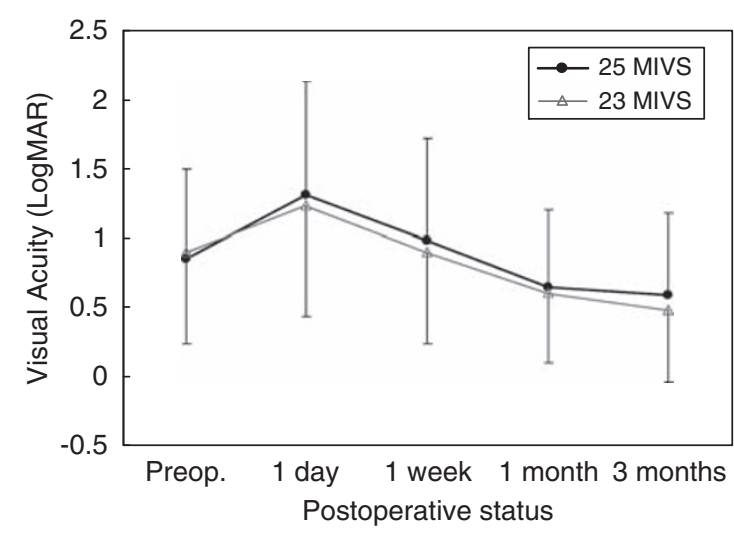

Figure 1 Mean visual acuity (LogMAR) \pm standard deviation at baseline and at 1 day, 1 week, and 1 and 3 months postoperatively. The respective results are $0.83 \pm 0.67$, $1.29 \pm 0.84,0.96 \pm 0.75,0.62 \pm 0.57$, and $0.57 \pm 0.59$ in group MIVS25, and $0.88 \pm 0.65,1.22 \pm 0.81,0.88 \pm 0.67,0.58 \pm 0.51$, and $0.46 \pm 0.53$ in group MIVS23. In both groups, visual acuity improved 1 and 3 months after surgery $(P=0.001$ and $P=0.017$, respectively, paired $t$-test). There was no statistically significant difference between the two groups at any point of follow-up.

Although the makers of each of two commercially available small gauges, 25 and 23 gauges, claim to offer the safest and best product, no studies have yet compared the 25- and 23-guage systems.

In this prospective study, we have shown that 25- and 23-gauge MIVS resulted in similar outcomes in terms of 

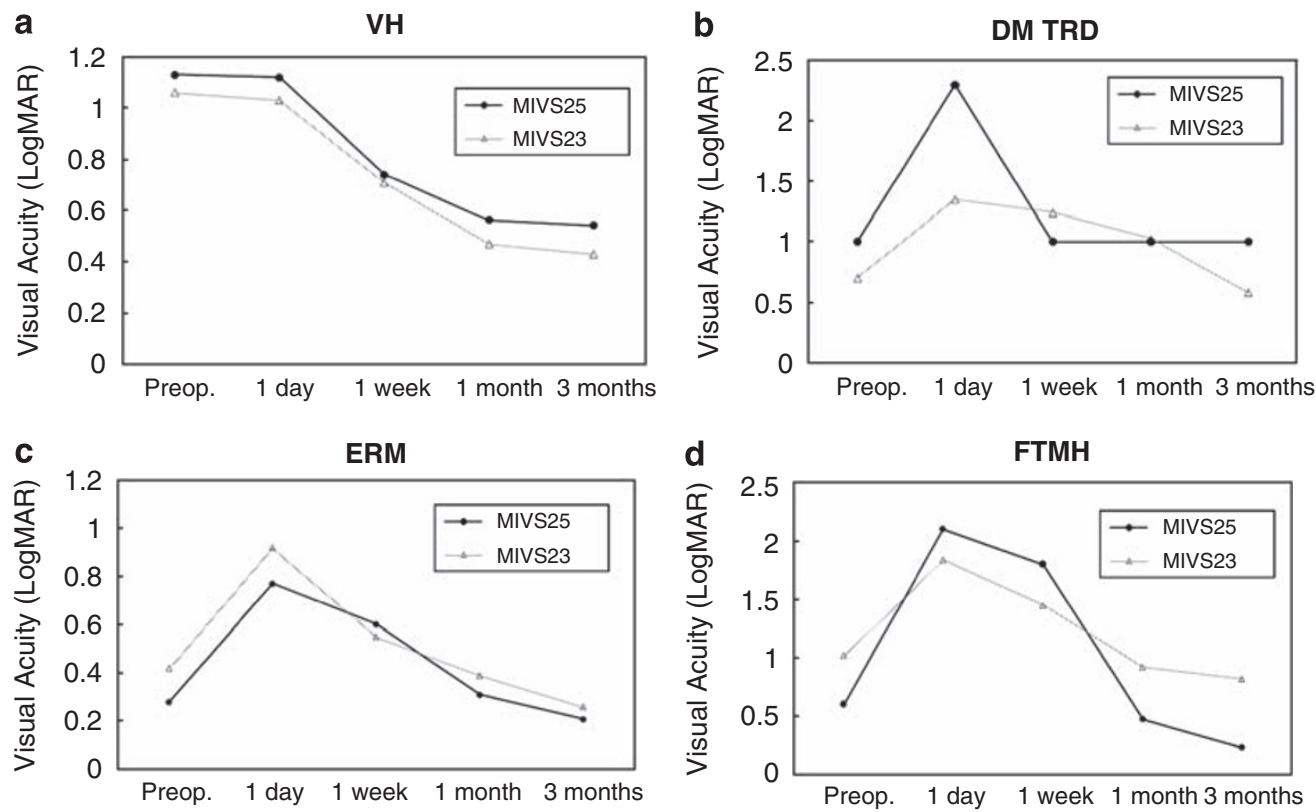

Figure 2 Visual acuity (LogMAR) at baseline and at 1 day, 1 week, and 1 and 3 months postoperatively in four subgroups of the MIVS23 and MIVS25 groups: (a) vitreous haemorrhage (VH), (b) diabetic traction retinal detachment (DMTRD), (c) epiretinal membrane (ERM), and (d) full-thickness macular hole (FTMH). In all subgroups, visual acuity improved 1 and 3 months after surgery. There was no statistically significant difference between the two groups at any point of follow-up.

Table 2 Conjunctival and corneal changes

\begin{tabular}{lccc}
\hline & MIVS25 $^{\mathrm{a}}$ & MIVS23 $^{\mathrm{a}}$ & P-value $^{\mathrm{b}}$ \\
\hline Postoperative 1 day & & & \\
$\quad$ Conjunctival hyperemia & $0.94 \pm 0.56$ & $1.03 \pm 0.53$ & 0.46 \\
Conjunctival oedema & $0.90 \pm 0.57$ & $1.02 \pm 0.56$ & 0.33 \\
SCH & $1.04 \pm 0.81$ & $1.14 \pm 0.76$ & 0.59 \\
Corneal oedema & $0.66 \pm 0.56$ & $0.67 \pm 0.47$ & 0.92 \\
& & & \\
Postoperative 1 week & & & \\
$\quad$ Conjunctival hyperemia & $0.42 \pm 0.28$ & $0.43 \pm 0.39$ & 0.94 \\
Conjunctival oedema & $0.16 \pm 0.28$ & $0.37 \pm 0.41$ & $0.03^{\mathrm{a}}$ \\
SCH & $0.42 \pm 0.49$ & $0.59 \pm 0.66$ & 0.38 \\
Corneal oedema & $0.12 \pm 0.26$ & $0.10 \pm 0.20$ & 0.78 \\
\hline
\end{tabular}

$\mathrm{SCH}$, subconjunctival haemorrhage.

Grading: none, 0 ; trace, +0.5 ; mild, +1 ; moderate, +2 ; severe, +3 . ${ }^{\mathrm{a}}$ Mean \pm SD.

${ }^{\mathrm{b} O n e-w a y ~ A N O V A}$ test.

efficacy and safety when used to treat various vitreoretinal diseases. First, the operation time was similar despite variations in cutter size and efficiency. We suspect this was partly because most of our patients had relatively uncomplicated vitreoretinal pathology and partly owing to the improved efficiency of the new 25-gauge cutter. Second, the rate of postoperative visual recovery did not differ significantly between the two groups. Although less postoperative inflammation, and consequent faster recovery, was expected in the 25-gauge group with smaller incisions and less BSS consumed, this
Table 3 Complications of MIVS

\begin{tabular}{lcc}
\hline & MIVS25 & MIVS23 \\
\hline $\begin{array}{l}\text { Intraoperative } \\
\quad \text { Conversion to 20 g }\end{array}$ & 0 & 0 \\
$\quad \begin{array}{l}\text { Suture of sclerotomy } \\
\quad \text { Retinal tear }\end{array}$ & 3 & 7 \\
Postoperative & 1 & 1 \\
$\quad \begin{array}{l}\text { Hypotony (postoperative 1 day) } \\
\text { (IOP <8 mm Hg) }\end{array}$ & 2 & 3 \\
$\quad \begin{array}{l}\text { Choroidal detachment } \\
\text { Wound leakage }\end{array}$ & 0 & 0 \\
$\begin{array}{l}\text { Recurrent VH } \\
\text { (postoperative 3 months) }\end{array}$ & 0 & 0 \\
\hline
\end{tabular}

IOP, intraocular pressure; $\mathrm{VH}$, vitreous haemorrhage.

was not the case. The results from each group were very similar, not only when all cases were compared, but also when each of four diagnostic subgroups was analysed. This is the main difference from earlier studies, which showed significantly faster visual recovery in either 25- or 23-gauge MIVS patients, compared with those receiving 20-gauge vitrectomy. Third, although the size of sclerotomy wounds was larger in the 23-gauge group than in 25-gauge patients, only a slight difference was seen in the postoperative status of both cornea and conjunctiva. Finally, no significant between-group differences in intraoperative or postoperative complications were identified. Minor differences 
between the 25- and 23-gauge groups were seen in the number of eyes that required suturing sclerotomy at the end of surgery and the number of cases of transient postoperative hypotony.

Small-gauge 25- or 23-gauge sutureless MIVS has evolved significantly, as a sutureless technique was first introduced in 2002. During the past 7 years, there have been remarkable advances in the development of vitreous cutters and microsurgical accessories. The first generation 25-gauge cutter (Bausch \& Lomb) was safe and efficient in the treatment of simpler vitrectomy cases. However, owing to limited cutting efficiency and the lack of necessary accessories such as illuminating probes or microscissors/forceps, the 25-gauge vitrectomy system failed to gain widespread acceptance among vitreoretinal surgeons. In 2003, DORC introduced the 23-gauge vitrectomy system with a stiffer cutter, which gave better cutting efficiency. However, the technical difficulty involved in the use of two-step-entry trocars was a drawback. More recently, Alcon introduced disposable, one-step, easy-entry, trocars for both the 25- and 23-gauge systems. With newer cutters, better illuminating probes, and good accessory instruments, microincision vitrectomy techniques are now rapidly expanding the indications for sutureless vitrectomy and improving outcomes in vitreoretinal surgery. Furthermore, increasing numbers of published reports describing clinical success have paved the way for further acceptance of such techniques by clinicians.

Changes in the standard vitrectomy technique have been well reflected in the results of Preferences and Trends surveys conducted by the American Society of Retinal Specialists during the past 5 years. In 2003, only $30 \%$ of surgeons had tried the (Bausch \& Lomb) 25-gauge system; of these, $9 \%$ had used the system in more than half of their cases. But in 2006, $>80 \%$ of surgeons had tried the 25-gauge systems of either or both of Bausch \& Lomb and Alcon, and $36 \%$ of surgeons had used the 25-gauge systems in more than half of their cases. When the Alcon 23-gauge system was first introduced in 2006, small-gauge surgeons began to split into two groups: 25- vs 23-gauge communities. In 2007, > 80 and $50 \%$ of surgeons had tried the 25- and 23-gauge systems, respectively, and the percentage of surgeons who had used 25- or 23-gauge approaches for more than half of their cases were 36 and $13 \%$, respectively. Only $20 \%$ of surgeons still used 20-gauge vitrectomy as their primary procedure.

As the above trend shows, most surgeons today, including those who are just beginning vitrectomy, have a strong interest in adapting small-gauge MIVS surgery as their primary standard procedure. It is thus important to identify and recommend the best MIVS system to a surgeon just starting to use MIVS. Theoretically, the 25-gauge system should be superior to the 23-gauge technique, as a smaller wound is created, leading to less risk of wound leakage and faster wound healing. However, the 23-gauge system has a better cutting efficiency, possibly resulting in a shorter operation time, but still offers the wound self-sealing feature.

Our data, however, show that neither technique was superior to the other in terms of surgical efficiency or anatomical/functional outcomes. Therefore, we can only suggest that any new vitreoretinal surgeon may choose MIVS of either gauge on the basis of his/her personal preference or the availability of allied instrumentation.

Our study has several limitations. First, the inclusion criteria for MIVS were limited to rather simple indications for vitrectomy. It may be speculated that the 23-gauge system would have been shown to be better than the 25-gauge technique if more advanced cases, such as those with diabetic traction retinal detachment of moderate degree or worse, cases of early proliferative vitreoretinopathy, or patients requiring silicone oil tamponade, were included. Second, a surgeon with a practice history different from that of the surgeon in this study might obtain a different outcome in a similar study; the surgeon in this study had more experience with the 25-gauge system than with the 23-gauge technique.

In conclusion, our prospective, randomized, comparative study showed that when inclusion criteria were limited to macular surgery and rather simpler vitreoretinal cases, the surgical outcomes and safety profiles were similar in the 25- and 23-gauge MIVS groups. Therefore, selection of small-gauge size may be made on the basis of a surgeon's personal experience or hospital circumstances.

\section{Conflict of interest}

The authors declare no conflict of interest.

\section{References}

1 Machemer R, Buettner H, Norton EW, Parel JM. Vitrectomy: a pars plana approach. Trans Am Acad Ophthalmol Otolaryngol 1971; 75: 813-820.

2 Fujii GY, de Juan Jr E, Humayun MS, Pieramici DJ, Chang TS, Awh C et al. A new 25-gauge instrument system for transconjunctival sutureless vitrectomy surgery. Ophthalmology 2002; 109: 1807-1813.

3 Fujii GY, de Juan Jr E, Humayun MS, Chang TS, Pieramici DJ, Barnes A et al. Initial experience using the transconjunctival sutureless vitrectomy system for vitreoretinal surgery. Ophthalmology 2002; 109: 1814-1820.

4 Ibarra MS, Hermel M, Prenner JL, Hassan TS. Longer-term outcomes of transconjunctival sutureless 25-gauge vitrectomy. Am J Ophthalmol 2005; 139: 831-836. 
5 Lakhanpal RR, Humayun MS, de Juan Jr E, Lim JI, Chong LP, Chang TS et al. Outcomes of 140 consecutive cases of 25-gauge transconjunctival surgery for posterior segment disease. Ophthalmology 2005; 112: 817-824.

6 Kellner L, Wimpissinger B, Stolba U, Brannath W, Binder S. 25-gauge vs 20-gauge system for pars plana vitrectomy: a prospective randomised clinical trial. Br J Ophthalmol 2007; 91: 945-948.

7 Yanyali A, Celik E, Horozoglu F, Oner S, Nohutcu AF. 25-gauge transconjunctival sutureless pars plana vitrectomy. Eur J Ophthalmol 2006; 16: 141-147.

8 Rizzo S, Genovesi-Ebert F, Murri S, Belting C, Vento A, Cresti $\mathrm{F}$ et al. 25-Gauge, sutureless vitrectomy and standard 20-gauge pars plana vitrectomy in idiopathic epiretinal membrane surgery: a comparative pilot study. Graefes Arch Clin Exp Ophthalmol 2006; 244: 472-479.

9 Shimada H, Nakashizuka H, Mori R, Mizutani Y, Hattori T. 25-gauge sclera tunnel transconjunctival vitrectomy. $A m \mathrm{~J}$ Ophthalmol 2006; 142: 871-873.

10 Ibarra MS, Hermel M, Prenner JL, Hassan TS. Longer-term outcomes of transconjunctival sutureless 25-gauge vitrectomy. Am J Ophthalmol 2005; 139: 831-836.

11 Lam DS, Yuen CY, Tam BS, Cheung BT, Chan WM. Sutureless vitrectomy surgery. Ophthalmology 2003; 110: 2428-2429.

12 Meyer CH, Rodrigues EB, Schmidt JC, Hörle S, Kroll P. Sutureless vitrectomy surgery. Ophthalmology 2003; 110: 2427-2428.

13 Eckardt C. Transconjunctival sutureless 23-gauge vitrectomy. Retina 2005; 25: 208-211.
14 Kadonosono K, Yamakawa T, Uchio E, Yanagi Y, Tamaki Y, Araie M. Comparison of visual function after epiretinal membrane removal by 20 -gauge and 25 -gauge vitrectomy. Am J Ophthalmol 2006; 142: 513-515.

15 Misra A, Ho-Yen G, Burton RL. 23-gauge sutureless vitrectomy and 20-gauge vitrectomy: a case series comparison. Eye 2009; 23: 1187-1191.

16 Okamoto F, Okamoto C, Sakata N, Hiratsuka K, Yamane N, Hiraoka $\mathrm{T}$ et al. Changes in corneal topography after 25-gauge transconjunctival sutureless vitrectomy vs after 20-gauge standard vitrectomy. Ophthalmology 2007; 114: 2138-2141.

17 Yang SJ, Yoon SY, Kim JG, Yoon YH. Transconjunctival sutureless vitrectomy for the treatment of vitreoretinal complications in patients with diabetes mellitus. Ophthalmic Surg Lasers Imaging 2009; 40 (in press).

18 Saika S, Tanaka T, Miyamoto T, Ohnishi Y. Surgical posterior vitreous detachment combined with gas/air tamponade for treating macular edema associated with branch retinal vein occlusion: retinal tomography and visual outcome. Graefe's Arch Clin Exp Ophthalmol 2001; 239: 729-732.

19 Barraquer RI, Alvarez de Toledo JP, Montané D, Escoto RM, Garcia Torres C, Bennani-Tazzi M. Fixed-dose combination of $0.1 \%$ diclofenac plus $0.3 \%$ tobramycin ophthalmic solution for inflammation after cataract surgery. Eur J Ophthalmol 1998; 8: 173-178.

20 Wimpissinger B, Kellner L, Brannath W, Krepler K, Stolba $\mathrm{U}$, Mihalics C et al. 23- vs 20-gauge system for pars plana vitrectomy: a prospective randomized clinical trial. $\mathrm{Br} J$ Ophthalmol 2008; 92: 1483-1487. 